\title{
Incidence and Prevalence of Atherosclerotic Renal Artery Stenosis (RAS) in Patients with Coronary Artery Disease (CAD)
}

\author{
AHMW Islam, S Munwar, S Talukder, AQM Reza \\ Dept. of Invasive \& Interventional Cardiology, Apollo Hospitals, Dhaka.
}

Keywords:
Renal artery
stenosis,
Coronary Artery
Disease

\begin{abstract}
:
Background: Aim of our present study was to evaluate the extent of Renal Artery Stenosis(RAS) in patients with Coronary Artery disease (CAD) in context of Bangladesh demographic distribution.

Methods: Total 100 patients with renal artery disease were randomized from a pool of 1200 patients who were underwent routine diagnostic coronary angiogram for the evaluation of extent of their $C A D$. Renal arteries were studied at the same time. Among the patients, Male: 64 and Female:36. Mean age were for Male:62yrs, for Female: 58 yrs. Associated CAD risk factors were Dyslipidemia, High Blood pressure, Diabetes Mellitus, Positive FH for CAD and Smoking.

Results: Our study results show 100 patients (12\% of the sample) had RAS lesion out of total 1200 patient who underwent routine CAG. Sex distribution is male 64 (64\%), Female 36 (36\%). Among the study group; 79 (79\%) were hypertensive; 57 (57\%) were Dyslipidemic, 54 (54\%) patients were Diabetic, 27 (27\%) were smoker (all male) and 18(18\%) were having positive FH for CAD. Female patients were more obese and developed CAD in advanced age (male: 53.5 Versus Female:64 yrs). We found that the Prevalence's of RAS is more in patients with TVD (43\%), followed by DVD (10\%) and SVD (10\%), Minor to mod CAD (14\%) and angiographically normal coronaries (23\%). Significant (>50\% Stenosis) Left renal artery stenosis were found in 27 patient and 23 had significant Right renal artery stenosis. Total 8 patents had significant both renal artery stenosis.

Conclusion: Renal artery stenosis is one of the most important peripheral vascular disease, needing to be diagnosed early and subsequent intervention to keep continuity of renal blood flow. In the present study, we found that the significant association of Renal Artery Stenosis in patients with $C A D$. Incidence of RAS is more in patients with TVD. Therefore, we recommend routine Renal angiogram during coronary angiography.
\end{abstract}

(Cardiovasc. j. 2010; 2(2) : 179-183)

\section{Introduction:}

Atherosclerotic renal artery stenosis(RAS) is a common anatomic finding in patients with atherosclerosis in other arterial circulation. ${ }^{1}$ There is also increase prevalence of RAS in patients with known or suspected coronary artery disease(CAD). ${ }^{2-4}$ RAS is associated with premature cardiovascular events like myocardial infarction, stroke or death, and double the risk of mortality in patient with $\mathrm{CAD}$ when revascularizations were performed. ${ }^{5}$ It is also a potentially correctable cause of hypertension, ischaemic nephropathy and loss of renal mass. Current endovascular therapies for RAS achieve high procedural success rates with excellent long-term patency rates, preservation of renal function and more manageable hypertension. Needless to mention, that in Bangladeshi perspective, we don't have an exact data on the incidence, prevalence and survival out come of patient with RAS associated with CAD and other co morbid renal condition. Therefore, we have carried out this study to assess the incidence of RAS in patients with CAD to reduced future mortality and morbidity.

\section{Methodology:}

A total of 1200 patients referred for cardiac catheterization because of suspected CAD were studied. Symptoms of coronary artery disease were recorded and classified according to $\mathrm{CCS}^{6}$ or NYHA. ${ }^{7}$ Blood samples including S. creatinine were measured before the procedure. All patients received Aspirin $300 \mathrm{mg} /$ day and Clopidegrol as a loading dose of $300 \mathrm{mg}$ prior to CAG. CAG was

Address of Correspondence: Dr. A H M Waliul Islam, Dept of Interventional and Invasive cardiology, Apollo Hospitals Dhaka, Bangladesh. 
done either via Femoral or Radial approach. After CAG, selective left and Right Renal arteriography was performed in the anterior-posterior projection with Iopamiro 370 at a rate of $20 \mathrm{ml} . \mathrm{S}-1$ to total $10-15 \mathrm{ml}$. An angiographically significant RAS was defined by narrowing of the lumen $>50 \%$ and high grade RAS as $>70 \%$ narrowing. ${ }^{4}$

In the studied population, subjects were required to meet at least one of the predefined selection criteria: 1) Severe atherosclerosis; 2) Severe or resistant hypertension 3) unexplained renal dysfunction with high serum creatinine; 4) acute pulmonary edema due to diastolic LV dysfunction. Data analysis: Data were presented as mean \pm SD.

\section{Results:}

Routine CAG and Renal arteriography was done in total 1200 patients. As shown in Table.1, total 100 patient, Male 64 and female: 36 were included in the study. Male were older than female (male $62 \pm 9$; Female: $58 \pm 10$ ). Female were more obese than male (BMI Male 24.5 \pm 3.4 ; Female $26.9 \pm$ 3.5). In the present study group, all smokers were male, thus increased the number of vascular risk factors more in male than female (Male: $2.47 \pm 1.1$; Female 2.38 \pm 0.9 ). Patients were arranged in three groups as left renal stenosis, right renal stenosis and both renal stenosis.

\section{Table-I}

Demographic profile of the study population

\begin{tabular}{lcc}
\hline & $\begin{array}{c}\text { Male } \\
(\text { mean } \pm \text { SD })\end{array}$ & $\begin{array}{c}\text { Female } \\
(\text { mean } \pm \text { SD) }\end{array}$ \\
\hline Number & 64 & 36 \\
Age $(\mathrm{yrs})$ & $62 \pm 9$ & $58 \pm 10$ \\
$\mathrm{BMI}\left(\mathrm{kg} / \mathrm{m}^{2}\right)$ & $24.5 \pm 3.4$ & $26.9 \pm 3.5$ \\
$\mathrm{SBP}(\mathrm{mmHg})$ & $135.7 \pm 20$ & $137.9 \pm 22.4$ \\
$\mathrm{DBP}(\mathrm{mmHg})$ & $80.1 \pm 8.6$ & $80.7 \pm 10.2$ \\
No.of Risk Factor & $2.47 \pm 1.14$ & $2.38 \pm 0.9$ \\
S. Creatinine( & $1.4 \pm 0.6$ & $1.3 \pm 0.5$ \\
\hline
\end{tabular}

RAS with $>50 \%$ stenosis were considered as significant renal stenosis. Table 2. shows the profile of patient with Right renal stenosis. Patient with $>50 \%$ stenosis are more older with average age $62 \mathrm{yrs}$, higher S. Creatinine $(1.5 \pm 0.9)$ and average \% of stenosis $(81.4 \pm 11.7)$ than $<50$ stenosis. As shown in Table.3, patient with $>50 \%$ left renal stenosis have higher systolic BP
(140.5 \pm 24.3$)$, higher S. Creatinine (1.54 \pm 0.9$)$ and higher average $\%$ stenosis $(75.4 \pm 11.6)$. Table 4 . shows the profile of patient with both renal stenosis of $>50$. S. Creatinine level was higher among the all groups $(1.9 \pm 1.4)$. As shown in Fig. 1 Atherosclerotic Risk factors in the studied population. Of them HTN 79\%, Dyslipidemia 57\%, DM 54\%, Smoking 27\%, FH 18\%.

\section{Table-II}

Profile of Right Renal Artery Stenosis

\begin{tabular}{lcc}
\hline & $<50 \%$ stenosis & $>50 \%$ stenosis \\
\hline Sex $(\mathrm{M} / \mathrm{F})$ & $(20 / 12)$ & $23(16 / 7)$ \\
Age $(\mathrm{yrs})$ & $59 \pm 7.9$ & $62 \pm 13$ \\
$\mathrm{BMI}\left(\mathrm{kg} / \mathrm{m}^{2}\right)$ & $25.9 \pm 4.1$ & $23.6 \pm 3.4$ \\
$\mathrm{SBP}(\mathrm{mmHg})$ & $138 \pm 21.9$ & $137.8 \pm 24.2$ \\
$\mathrm{DBP}(\mathrm{mmHg})$ & $80.5 \pm 9.9$ & $80.5 \pm 7.7$ \\
No.of Risk Factor & $2.57 \pm 1.17$ & $2.4 .3 \pm 1.1$ \\
S. Creatinine & $1.3 \pm 0.4$ & $1.5 \pm 0.9$ \\
Average \% of & $29.5 \pm 8.3$ & $81.4 \pm 11.7$ \\
Stenosis & & \\
\hline
\end{tabular}

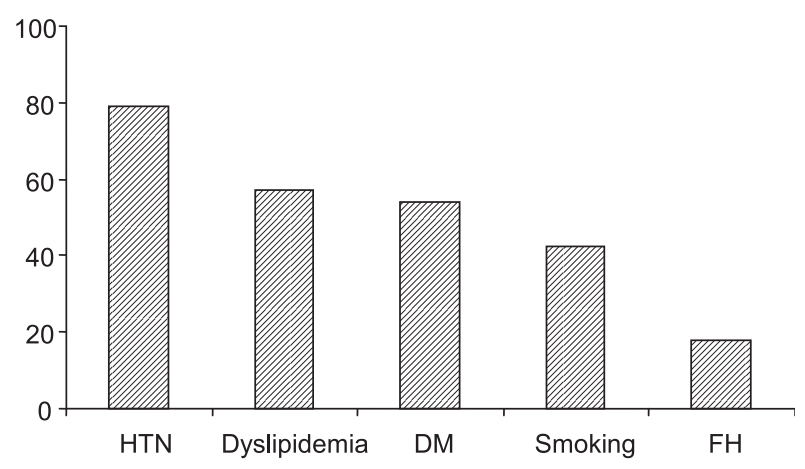

Fig.-1: Shows percent distribution of CAD risk factors

Table-III

Profile of Left Renal Artery Stenosis

\begin{tabular}{lcc}
\hline & $<50 \%$ stenosis & $>50 \%$ stenosis \\
\hline Sex $(\mathrm{M} / \mathrm{F})$ & $(34 / 17)$ & $(14 / 13)$ \\
Age $(\mathrm{yrs})$ & $61 \pm 8.5$ & $62 \pm 12$ \\
$\mathrm{BMI}\left(\mathrm{kg} / \mathrm{m}^{2}\right)$ & $25.3 \pm 3.4$ & $24.4 \pm 2.9$ \\
$\mathrm{SBP}(\mathrm{mmHg})$ & $134.7 \pm 21.2$ & $140.5 \pm 24.3$ \\
$\mathrm{DBP}(\mathrm{mmHg})$ & $79.9 \pm 8.9$ & $80.2 \pm 10.4$ \\
No.of Risk Factor & $2.24 \pm 1.0$ & $2.48 \pm 0.96$ \\
S. Creatinine & $1.24 \pm 0.4$ & $1.54 \pm 0.9$ \\
Average \% of & $33.3 \pm 10.9$ & $75.4 \pm 11.6$ \\
Stenosis & & \\
\hline
\end{tabular}


Table-IV

Profile of Significant Both Renal stenosis

\begin{tabular}{lc}
\hline & $>50 \%$ stenosis \\
\hline Sex $(\mathrm{M} / \mathrm{F})$ & $8(6 / 2)$ \\
Age $(\mathrm{yrs})$ & $62 \pm 17$ \\
$\mathrm{BMI}\left(\mathrm{kg} / \mathrm{m}^{2}\right)$ & $24.2 \pm 1.6$ \\
$\mathrm{SBP}(\mathrm{mmHg})$ & $130.8 \pm 32.9$ \\
DBP(mmHg) & $80.8 \pm 6.6$ \\
No.of Risk Factor & $2.33 \pm 0.8$ \\
S. Creatinine( & $1.9 \pm 1.4$ \\
Avg \% of Stenosis: Lt: & $83.12 \pm 10.9$ \\
& $83.1 \pm 7.5$ \\
\hline
\end{tabular}

Table-V

Percent distribution of CAD in Pt with Left Renal Stenosis

\begin{tabular}{lcc}
\hline & $<50 \%$ stenosis & $>50 \%$ stenosis \\
\hline LM & $3(5.8 \%)$ & $3(11.1 \%)$ \\
TVD & $24(47 \%)$ & $14(51.2 \%)$ \\
DVD & $5(9.8 \%)$ & $3(11 \%)$ \\
SVD & $9(17.6 \%)$ & $2(7.4 \%)$ \\
Minor CAD & $5(9.8 \%)$ & $2(7.4 \%)$ \\
Normal Coronaries & $5(9.8 \%)$ & $4(14.8 \%)$ \\
\hline
\end{tabular}

Table-VI

Percent distribution of CAD in Pt with Right Renal Stenosis

\begin{tabular}{lcc}
\hline & $<50 \%$ stenosis & $>50 \%$ stenosis \\
\hline LM & $4(12.5 \%)$ & $3(13 \%)$ \\
TVD & $14(43.7 \%)$ & $12(52.2 \%)$ \\
DVD & $3(9.4 \%)$ & \\
$1(4.3 \%)$ & & \\
SVD & $1(3.1 \%)$ & $3(13 \%)$ \\
Minor CAD & $8(25 \%)$ & $1(4.3 \%)$ \\
Normal Coronaries & $5(15.6 \%)$ & $3(13 \%)$ \\
\hline
\end{tabular}

Fig. 2 shows the percent distribution of CAD in patient with Renal artery stenosis. TVD 43\%, DVD $10 \%$, SVD $10 \%$, non significant CAD $14 \%$, angiographically normal coronaries $23 \%$. Total 9 patients had LM stem disease in association with TVD in 8 and SVD in 1 patient. Table 5 . and Table 6 shows the distribution of CAD in patient with Left and Right renal artery stenosis. We found that, significant renal artery stenosis were higher in patient with TVD (Left: 14 (51\%), Right: 12(52\%).

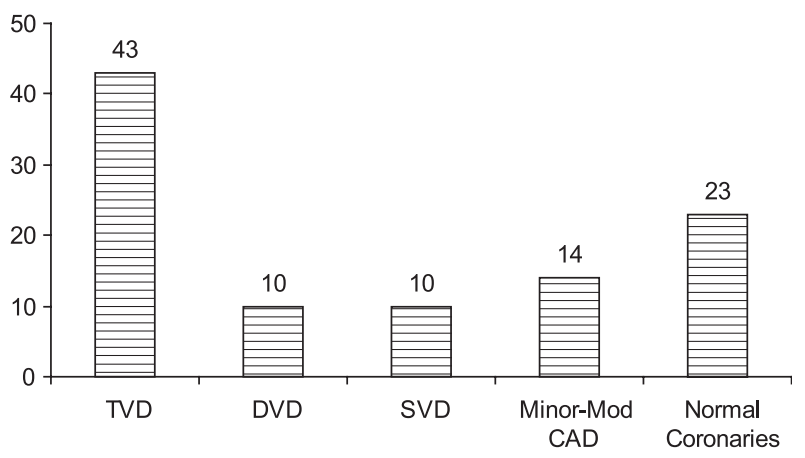

Fig.-2: Coronary Angiographic findings in the studied population

\section{Discussion:}

Renal artery stenos (RAS) often leads to progressive renal artery occlusion and constitutes a potentially correctable cause of hypertension. ${ }^{8-9}$ Patients with $>50 \%$ RAS has a higher rate of myocardial infarction or require cardiac revasularization. ${ }^{5,10}$ RAS often remains unrecognized as there is no specific RAS symptoms. ${ }^{3,11-12}$

To the best of our knowledge, this is the first study in Bangladesh to find out the incidence of RAS in patient with CAD. We tried to find out the percentage of distribution of RAS in patients going for routine diagnostic $\mathrm{CAG}$, underlying risk factors and subsequent treatment modality. In the present population, we found that significant RAS (>50\%) is associated with higher incidence of TVD. Thus, routine Renal angiography in patients with TVD is definitely helpful and as well life saving by performing Renal artery stenting (PTA) prior to CABG or multivessel PCI. This could prevent or worsen renal failure after CABG and or PCI.

The incidence of renal artery stenosis and CAD in patients undergoing coronary angiography in our patient population is $12 \%$, very much similar to other studies ${ }^{2,13}$.

The clinical diagnosis of atherosclerotic renal artery stenosis remains problematic. In contrast to myocardial ischaemia, the pathophysiologic manifestations of RAS (Hypertension, renal dysfunction, and acute left ventricular failure) are non specific. Contrast angiography is a standard criteria for RAS. It is readily performed in combination with coronary angiography. Correlation of coronary disease burden and RAS is already established. ${ }^{14}$ Although aortography 
does add cost to coronary angiographic procedure, there is good evidence that the addition of abdominal aortography in patient with baseline serum creatinine $<2.0 \mathrm{mg} / \mathrm{dl}$ is not associated with an increase in procedure-related morbidity or mortality. ${ }^{15}$ Patients at increased risk of contrast nephropathy should be pretreated with $\mathrm{N}$ acetylcysteine and receive vigorous hydration before administration of low-osmolar contrast. ${ }^{16}$ Non-invasive procedures like duplex ultrasonography, magnetic resonance arteriography or computer assisted tomography can be routinely used to diagnose renovascular pathology before diagnostic CAG. However, some of the high risk patient for RAS such as multivessel $\mathrm{CAD}$, unexplained renal dysfunction or pulmonary oedema, who are potential candidate for renal revascularization, will present for urgent CAG without having had the opportunity for noninvasive screening for RAS.

It is well known that the atherosclerosis accounts for 90 percent of cases of renal-artery stenosis ${ }^{9}$. The prevalence of atherosclerotic renal-artery stenosis increases with age, particularly in patients with diabetes, aortoiliac occlusive disease, coronary artery disease, or hypertension. Renal-artery stenosis may occur alone (isolated anatomical renal-artery stenosis) or in association with hypertension, renal insufficiency (ischemic nephropathy), or both. ${ }^{17}$ We found that high blood pressure (HTN) is an important risk factors for the development of renal artery stenosis (RAS) followed by Dyslipidemia, DM, FH and smoking.

Routing screening renal angiogram is helpful for the patients to screen any RAS and planning of further treatment. Also, it does help incidental findings of undetected renal causes of hypertension. In the present study, we found $12 \%$ patient with CAD has Mild to moderate significant RAS.

In the present study, we also found the association of significant RAS with raised serum creatinine (Table 3), specially higher level in the significant bilateral renal artery stenosis (Table 4). Thereby, serum creatinine could be an important predictor of significant renal artery stenosis in patients with CAD.

The increased prevalence of RAS in patients with coronary artery disease and the ultimate poor prognosis supports a strategy of increased awareness of this disease process and a need to identify RAS as early as possible.

We found that the renal artery stenosis (RAS) were present in significant number of patients with coronary artery disease $(\mathrm{CAD})$ either in the left or right renal artery and in certain patients both renal arteries were affected.

\section{Study Limitation:}

Our present study was carried out from the patient population, whom CAG was done for their chest pain complaint. Healthy group of patient were not included in this study.

\section{Conclusion:}

We conclude that it is reasonable to perform a screening renal arteriography at the time of CAG in patients at increased risk of RAS and who are candidates for revascularization ${ }^{16}$. Specially, in patient with significant $\mathrm{CAD}$ with triple vessel disease (TVD) and DVD with or without LM stem disease and elevated serum creatinine, hypertension, flushing pulmonary edema, abdominal bruit. ${ }^{17}$ This will reduce post CABG or PCI renal complications. Thus, could also help in preventing renal failure or progression of renal function impairment by early intervention.

Renal artery stenosis is prevalent in a significant proportion of patients undergoing cardiac catheterization for suspected coronary artery disease in Bangladeshi patient perspectives. The number of coronary artery disease with significant stenotic lesion is the most powerful predictor of associated significant renal artery stenosis. ${ }^{2-3,} 8,18$

\section{References:}

1. Scoble JE. Atherosclerotic Nephropathy. Kidney Intl Suppl 1999;71:S106-9.

2. Jean Wj, al-Bitar I, Wwicke DL et al. High incidence of renal artery stenosis in patients with coronary artery disease. Cath cadiovasc Diagn 1994;32:8-10.

3. Weber-Mezel D, Kotanko P, Schumacher M et al. Coronary anatomy predicts presence or absence of renal artery stenosis. Eur Heart $J$ 2002; 23::1684-1691.

4. Harding MB, Smith LR, Himmelstein SI et al. Renal artery stenosis: Prevalence and associates risk factors in patients undergoing routine cardiac catheterization. J Am Soc Nephrol 1992;2:1608-16.

5. Conlon PJ, Little MA, Pipper K et al. Severity of renal vascular disease predicts mortality in patients 
undergoing coronary angiography. Kidney Int 2001; 60:1490-1497.

6. Campeau L. Grading of angina pectoris. Circulation 1970; 54: 522-3.

7. Goldman L, Hashimoto B, Cook EF et al. Comparative reproducibility and validity of systems for assessing cardiovascular functional class: advantages of a new specific activity scale. Circulation 1981;64:1227-34.

8. Crowely JJ, Santos RM, Peter RH et al. Progression of renal artery stenosis in patients undergoing cardiac catheterization. Am Heart J 1998;136:913-918.

9. Caps MT, Perisinotto C, Zierler RE et al. Prospective study of atherosclerotic disease progression in the renal artery. Circulation 1998;98:2866-2872.

10. Edwards MS, Craven TE, Burke GL et al. Renovascular disease \& the risk of adverse coronary events in the elderly. Arch Internal Med 2005;165:2107-2137.

11. Aquel RA, Zoghbi GJ, Baldwin SA et al. Prevalence of renal artery stenosis in high risk veterans referred for card catheterization. J Hypertensions 2003; 21:11571162.

12. Park S, Jung JH, Seo HS et al. The prevalence and clinical predictors of atherosclerotic renal artery stenosis in patients undergoing coronary angiography.
13. Yamashita T, Ito F, Iwakiri N et al. Prevalence and predictors of renal artery stenosis in patient undergoing cardiac catheterization. Hypertension Res 2002; 25:5537 .

14. Buller CE, Nogareda JG, Ramanathan K et al. The profile of cardiac patients with renal artery stenosis. $J$ Am Coll Card 2004;43:1606-13.

15. Rihal CS, Textor SC, Breen JF et al. Incidental renal artery stenosis among a prospective cohort of hypertensive patients undergoing coronary angiography. Mayo clin Proc. 2002:77:309-316.

16. Hirsc AT, Haskal ZJ, Hertzer NR et al. ACC/AHA 2005 practice guidelines for the management of patients with peripheral arterial disease. Circulation.2006;113:e463e654.

17. Rigatelli G. Aortoiliac angiography during coronary angiography detects significant occlusion of aortoiliac and renal artery atherosclerosis in patients with coronary atherosclerosis. Inter J Cardiovascular Imag 2004; 20:299-303.

18. Vetrovec GW, Landwehr DM, Edwards VL. Incident of renal artery stenosis in hypertensive patients undergoing coronary angiography. J Interv Cardiol 1989;2:69-76. 